\title{
Evolution of Evaporating Black Holes in a Higher Dimensional Inflationary Universe.
}

\author{
Manasse R. Mbonye \\ Physics Department. University of Michigan. Ann Arbor. Michigan \\ 48109
}

\begin{abstract}
Spherically symmetric Black Holes of the Vaidya type are examined in an asymptotically de Sitter, higher dimensional spacetime. The various horizons are identified and located. The structure and dynamics of such horizons are studied.
\end{abstract}

\section{INTRODUCTION}

Several solutions to the Einstein equations of localized sources in higher dimensions have been obtained in the recent years. This includes the higher dimensional generalizations of the Schwarzschild and the Reisner-Nordstrom solutions [1], the Kerr solution [2] and the Vaidya solution [3]. Recently the metric of a radiating black hole in a de Sitter background, that is a generalization of the Mallett [4] metric, has been written down [5]. In the present work our aim is to demonstrate that the dynamics of a radiating black hole in a higher dimensional cosmological background can be sensibly discussed. First, we seek to identify and locate the various horizons. Then we study the structures and discuss the dynamics of such horizons. It is shown, at each stage, that all the results we obtain reduce to the well known Mallett [6] results as we go down to four dimensions.

In Section I we introduce the working metric. In Section II we derive equations for the horizons. We solve these equations and use the solutions to identify and locate the various horizons in the problem. In Section III we take up the issue of the structure of such horizons and study their dynamics. In Section IV we conclude the discussion. 


\section{THE METRIC}

We wish to consider a radiating black hole introduced in an $\mathrm{N}$ dimensional de Sitter space-time. In advanced time, comoving, coordinates the line element is [5],

$d s^{2}=-\left[1-\frac{2 G_{N} m(v)}{n r^{n}}-\frac{2 \Lambda}{(n+1)(n+2)} r^{2}\right] d v^{2}+2 d v d r+d \Omega_{n+1}^{2}$,

where $n=N-3, m(v)$, the mass, is a monotonically decreasing function of the advanced time coordinate $v, G_{N}$ is the $\mathrm{N}$-dimensional gravitational constant, $\Lambda$ is the cosmological constant and $d \Omega_{n+1}^{2}=d \theta_{1}^{2}+\sin ^{2} \theta_{1} d \theta_{2}^{2}+\ldots+$ $\sin ^{2} \theta_{1} \sin ^{2} \theta_{2} \ldots \sin ^{2} \theta_{n} d \theta_{n+1}^{2}$ is the line element on the $(n+1)$-sphere. The Luminosity $L_{0}=-\frac{d m}{d v}<1$, is measured in regions where $\frac{d}{d v}$ is time-like.

One can introduce a basis of $N$ vectors at every point in this spacetime. Two such vectors $\beta_{a}$ and $l_{a}$ that span the radial-temporal subspace are $\beta_{a}=\delta_{a}^{v}$ and $l_{a}=$ $-\frac{1}{2}\left[1-\frac{2 G_{N m(v)}}{n r^{n}}-\frac{2 \Lambda}{(n+1)(n+2)} r^{2}\right] \delta_{a}^{v}+\delta_{a}^{r}$. The rest of the $(N-2)$ vectors are defined on the $(n+1)$-sphere and induce on the latter a tensor field $\gamma_{a b}$ of the form

$\gamma_{a b}=r^{2}\left(\delta_{a}^{\theta_{1}} \delta_{b}^{\theta_{1}}+\sin ^{2} \theta_{1} \delta_{a}^{\theta_{2}} \delta_{b}^{\theta_{2}}+\ldots+\sin ^{2} \theta_{1} \sin ^{2} \theta_{2} . . \sin ^{2} \theta_{n} \delta_{a}^{\theta_{n+1}} \delta_{b}^{\theta_{n+1}}\right)$.

The vectors satisfy $\beta_{a} \beta^{a}=l_{a} l^{a}=0, \gamma_{a b} k^{b}=\gamma_{a b} l^{b}=0, \beta_{a} l^{a}=-1$.

\section{LOCATION OF THE HORIZONS}

The structure and dynamics of horizons of such non-static metrics can be approached from the non-perturbative description of deformation of relativistic membranes. The quantities that characterize how a variation in the symmetry of a membrane evolves are the expansion rate, $\theta$, the shear rate, $\sigma$, and the vorticity (twist), $\omega$. They obey the Rachaudhuri [7] equation,

$\frac{d \theta}{d v}=\kappa \theta-\left(\gamma_{c}^{c}\right)^{-1} \theta^{2}-\sigma_{a b} \sigma^{a b}+\omega_{a b} \omega^{a b}-R_{a b} l^{a} l^{b}$

where $R_{a b}$ is the $\mathrm{N}$-dimensional Ricci tensor, $\gamma_{c}^{c}$ is the trace of the projection tensor for null geodesics and $\kappa$ is to be identified as the surface gravity.

Spherically symmetric irrotational spacetimes, such as under consideration, are vorticity and the shear free. The structure and dynamics of the horizons are then only dependent on the expansion, $\theta$. Following York [8] we note that to $O\left(L_{0}\right)$ the evolution of an apparent horizon $(A H)$ is to satisfy the requirement that $\theta \simeq 0$, while that of an event horizons $(E H)$ is to satisfy the requirement that $\frac{d \theta}{d v} \simeq 0$.

\section{The Apparent Horizons}

In our basis the expansion $\theta$ can be written as

$$
\theta=\gamma^{a b} \nabla_{a} l_{b}
$$


From eq. (2.2) and the York condition $\theta \simeq 0$ we find that the $(A H s)$ satisfy,

$r^{n+2}-\frac{(n+1)(n+2)}{2 \Lambda} r^{n}+\frac{(n+1)(n+2)}{n} \frac{G_{N} m(v)}{\Lambda}=0$.

While eq. (2.3) admits no simple solutions it is possible to cast it in a form for which approximate solutions are reasonable and justifiable. To this end we set $r=k(1-\xi)$ where $k=\sqrt{\frac{(n+1)(n+2)}{2 \Lambda}}$ to transform eq. (2.3) to the form

$\xi(2-\xi)(1-\xi)^{n}-\beta(n)=0$.

where $\beta(n)=\frac{2 G_{N m(v)}}{n}\left[\frac{2 \Lambda}{(n+1)(n+2)}\right]^{\frac{n}{2}}$. In our model $\Lambda>0$ and so $\beta(n)>0$ so that for real and positive $r$ values, $0<\xi<1$ and $0<\beta<1$. This justifies seeking lower order solutions by expanding the expression $(1-\xi)^{n}$ in the first term of equation (2.4). Thus to $O\left(\xi^{4}\right)$ we find

$\xi^{4}+a \xi^{3}+b \xi^{2}+c \xi+d=0$

where $a=-\frac{6 n}{(n-1)(2 n-1)}, \quad b=\frac{6(2 n+1)}{n(n-1)(2 n-1)}, \quad c=-\frac{12}{n(n-1)(2 n-1)}$, and $d=$ $\frac{6}{n(n-1)(2 n-1)} \beta(n)$. We should mention that this expansion is strictly valid for $n>2$, although the solutions have the right limits when $n=1$. The solutions for the 5 -dimensional $(n=2)$ case are exact. In general this approximation is good in the limit $\xi \rightarrow 0$.

Of the four solutions for eq. (2.5) the two physically interesting ones for our purposes are

$\xi_{ \pm}=-\frac{1}{4} a+\frac{1}{2} R(p, \varphi) \pm \frac{1}{2} D(p, \varphi)$,

where $R=\sqrt{\frac{a^{2}}{4}-b+\left(2 \sqrt{\frac{-p}{3}}\right) \cos \frac{1}{3} \varphi}, \quad D=\sqrt{\frac{3 a^{2}}{4}-R^{2}(p, \varphi)-2 b+\frac{4 a b-8 c-a^{3}}{4 R(p, \varphi)}}$ and where $\left(\frac{\pi}{2}<\varphi<\pi\right)=\arccos \left(\frac{3 q}{2 \sqrt{\frac{p^{3}}{3}}}\right), p=\frac{1}{3}\left[\left(3 a c-b^{2}\right)-12 d\right]$ and $q=$ $\frac{1}{27}\left[9 a b c-2 b^{3}-27 c^{2}-9\left(3 a^{2}+b\right) d\right]$.

Recalling that $r=k(1-\xi)$, the solutions above give

$r_{A H}^{ \pm}(v) \simeq k\left\{1 \pm \frac{1}{4}[2 D(p, \varphi) \mp 2 R(p, \varphi) \pm a]\right\}$.

In the limit $n \rightarrow 1$ one recovers the well known solutions [6]. Thus

$\lim _{n=1} r_{A H}^{ \pm}(v)= \pm\left(\frac{2}{\sqrt{\Lambda}}\right) \cos \frac{1}{3} \Psi_{ \pm}$

where $\left(\frac{\pi}{2}<\Psi_{-}(v)<\pi\right)=\arccos [-3 m(v) \sqrt{\Lambda}]$ and $\Psi_{+}=\left(\Psi_{-}+\pi\right)$. 
Consequently, we identify the loci of $r_{A H}^{-}(v)$ and $r_{A H}^{+}(v)$ in equations $(2.7)$ as the black hole and de sitter apparent horizons $\left(A H^{-}\right)$and $\left(A H^{+}\right)$, respectively, for a radiating black hole in an $\mathrm{N}$-dimensional background with a cosmological constant.

\section{The Event Horizons}

The event horizons are null surfaces. To $O\left(L_{0}\right)$ the evolution of these surfaces can be determined by applying to eq. (2.2) the second of the York conditions, $\left.\frac{d \theta}{d v}\right|_{E H} \simeq 0$. One finds that the event horizons $(E H s)$ in this problem satisfy

$r^{n+2}-\frac{(n+1)(n+2)}{2 \Lambda} r^{n}+\frac{(n+1)(n+2)}{n} \frac{G_{N} m^{*}(v)}{\Lambda}=0$.

where $m^{*}(v)$ is some effective mass given by $m^{*}(v)=m(v)-\frac{L_{0}}{\kappa}$

Eq. (2.9) is exactly of the same form as its counterpart equation (2.3) with the mass $m(v)$ replaced by the effective mass $m^{*}(v)$. Hence we can immediately write the solutions to $(2.9)$ as

$r_{E H}^{ \pm}(v) \simeq k\left\{1 \pm \frac{1}{4}\left[2 D^{*}(p, \varphi) \mp 2 R^{*}(p, \varphi) \pm a^{*}\right]\right\}$,

where $*$ means $m(v) \rightarrow m^{*}(v)=m(v)-\frac{L_{0}}{-\kappa}$ and $\frac{1}{2} \pi<\varphi^{*}<\pi$.

In the limit $n \rightarrow 1$ one recovers the well known solutions [6]. Thus

$\lim _{n=1} r_{E H}^{ \pm}(v)= \pm\left(\frac{2}{\sqrt{\Lambda}}\right) \cos \frac{1}{3} \Psi_{( \pm)}^{*}$,

where $\left(\frac{\pi}{2}<\Psi_{(-)}^{*}(v)<\pi\right)=\arccos \left[-3 m^{*}(v) \sqrt{\Lambda}\right]$ and $\Psi_{(+)}^{*}=\left(\Psi_{(-)}^{*}+\pi\right)$

Consequently, we identify the loci of $r_{A H}^{-}(v)$ and $r_{A H}^{+}(v)$ in eq. (2.10) as the black hole and de sitter event horizons, i.e. $\left(E H^{-}\right)$and $\left(E H^{+}\right)$respectively, for a radiating black hole in an $\mathrm{N}$-dimensional background with a cosmological constant.

The ordering of the horizons can now be made. One finds from our results that $E H^{-}<A H^{-}<A H^{+}<E H^{+}$.

\section{STRUCTURE AND DYNAMICS OF THE HORIZONS}

\section{Structure of the Apparent Horizons}

At the apparent horizons the expansion, $\theta$, vanishes. One then finds that eq. (1.1) will, along with eqs. (2.7), induce on the surfaces $r_{A H}^{-}$and $r_{A H}^{+}$metrics of the form

$\left.d s^{2}\right|_{r=r_{A H}^{\ddagger}}=\mp \frac{k}{2} \alpha_{ \pm}(p, \varphi)\left[\rho \frac{\sin \frac{1}{3} \varphi}{\sin \varphi}+\sigma \cos \frac{1}{3} \varphi\right] \frac{d m}{d v} d v^{2}+d \Omega_{n+1}^{2}$, 
where $\alpha_{ \pm}(p, \varphi)=(D R)^{-1}\left[2 R+\frac{\left(4 a c-8 c-a^{3}\right)}{4 R} \pm 1\right], \quad \rho=\frac{1}{3} \sqrt{\frac{-p}{3}} \frac{d}{d m}\left(\frac{3 q}{2 \sqrt{\frac{-p^{3}}{3}}}\right)$ and $\sigma=\frac{2 d}{m(v) \sqrt{-3 p}}$. Noting that $\alpha, \rho$ and $\alpha_{ \pm}$are all positive quantities then for $\frac{\pi}{2}<\varphi<\pi, \frac{d m}{d v}<0$ contributes the only negative quantity in eqs. (3.1). We conclude, on this basis that the apparent horizon surface $r_{A H}^{-}(v)$ of an evaporating black hole in a higher dimensional de Sitter spacetime is timelike while the associated cosmological apparent horizon $r_{A H}^{+}$is spacelike.

In the limit $n \rightarrow 1$, one finds that

$\left.d s^{2}\right|_{r=r_{A H}^{ \pm}}= \pm \frac{4 \sin \left(\frac{1}{3} \Psi_{ \pm}\right)}{\sin \Psi} \frac{d m}{d v} d v^{2}+d \Omega_{n+1}^{2}$

Eqs. (3.2) are the known results [6] for the four dimensional case.

\section{The dynamics of the Horizons}

One can rewrite equations (3.1) in the form

$\left.d s^{2}\right|_{r=r_{A H}^{ \pm}} \simeq \pm 2 L_{0} \Gamma_{(A H) n}^{ \pm} d v^{2}+d \Omega_{n+1}^{2}$

where $\Gamma_{(A H) n}^{ \pm}=\frac{k}{2} \alpha_{( \pm)}(p, \varphi)\left[\rho \frac{\sin \frac{1}{3} \varphi}{\sin \varphi}+\sigma \cos \frac{1}{3} \varphi\right]$. We see then that to $O\left(L_{0}\right)$ the apparent horizons $r_{A H}^{ \pm}$move with velocities given by

$$
\frac{d r_{A H}^{ \pm}}{d v} \simeq \pm 2 L_{0} \Gamma_{(A H) n}^{ \pm}
$$

Similarly the motion of the event horizons $r_{E H}^{ \pm}$can be deduced from eqs. (2.9). One finds that the velocities of these surfaces are given by

$$
\frac{d r_{E H}^{ \pm}}{d v} \simeq \pm 2 L_{0} \Gamma_{(E H) n}^{ \pm}
$$

where the $\Gamma_{(E H) n}^{ \pm}$are obtained by applying to $\Gamma_{(A H) n}^{ \pm}$the transformation $m(v) \rightarrow$ $m^{*}(v)=m(v)-\frac{L_{0}}{\kappa}$ that turns $A H$ quantities to $E H$ quantities. For the range $\left(\frac{1}{2} \pi<\varphi, \varphi^{*}<\pi\right)$ considered $\Gamma_{(A H) n}^{ \pm}$and $\Gamma_{(E H) n}^{ \pm}$are positive so that for the observer at $r_{A H}^{-}<r<r_{A H}^{+}$both $E H^{-}$and $A H^{-}$move with negative velocities $-2 L_{0} \Gamma_{(E H) n}^{-}$ and $-2 L_{0} \Gamma_{(A H) n}^{-}$, respectively. Such motion represents, in each case, a contraction of the respective black hole horizon. Conversely the cosmological horizons $\mathrm{EH}^{+}$and $A H^{+}$are observed to expand at velocities $2 L_{0} \Gamma_{(E H) n}^{+}$and $2 L_{0} \Gamma_{(A H) n}^{+}$, respectively.

As $(n \rightarrow 1)$ one recovers the known results [6] for the four dimensional case, and in this limit as $\Lambda \rightarrow 0$, we recover from the equations the standard relations $\lim _{\substack{n-1 \\ \Lambda \rightarrow 0}} \frac{d r^{-} H}{d v}=-2 L_{0}$ and $\lim _{\substack{n-1 \\ \Lambda \rightarrow 0}} \frac{d r_{E H}^{-}}{d v}=-2 L_{0}$. 


\section{CONCLUSION}

In this discussion we have examined black holes of the Vaidya type in an spatially flat higher dimensional spacetime with a cosmological constant. We find four horizons identified as the event horizon, $E H^{-}$and the apparent horizon $A H^{-}$for the black hole and their cosmological counterparts, $E H^{+}$and $A H^{+}$, respectively. We have, to good order of accuracy, located these horizons and deduced both their structure and dynamics. Our results reduce to the known ones, under various limits, including the four dimensional case [6]. It is seen then that the problem of the dynamics of a radiating blackhole in a higher dimensional cosmological background can be sensibly discussed.

An application of our results to the Hawking radiation problem will be the topic of a future discussion, elsewhere.

\section{REFERENCES}

1. A. Chados and S. Detweiler, Gen Relativ. Gravit. 14, 879 (1982); G. W. Gibbons and D. L. Wiltshire, Ann. Phys. 167, 201 (1986).

2. R. C. Myers and M. J. Perry, Ann. Phys. (NY) 172, 304 (1986); P. O. Mazur, J. Math. Phys., 28, 406 (1987).

3. B. R. Iyer and C. V. Vishveshwara, Pramana J. Phys., 32, 749 (1989).

4. R. L. Mallett, Phys. Rev. D 31, 416 (1985).

5. L. K. Patel and L S. Desai, Pramana J. Phys. 48, 819 (1997); Zhen-Qiang Tan and You-Gen Shen, Il Nuovo Cimento 113 B, 339 (1998).

6. R. L. Mallett, Phys. Rev D 33, 2201 (1986).

7. A. Raychaudhuri, Phys. Rev. 98, 1123 (1955); B. Carter, General Relativity, (edited by S. W. Hawking and I. Isreal) Cambridge Univ. Press, Cambrige, UK (1979)

8. J. W. York, Jr., in Quantum Theory of Gravity: Essays in Honor of the Sixtieth Birthday of Bryce S. DeWitt, edited by S. Christensen (Hilger, Bristol, 1984). 